\title{
Cancer Pain: Incompletely Assessed, Inadequately Treated
}

\author{
Pankaj Vats ${ }^{1}$ and Virender Suhag ${ }^{2 *}$ \\ ${ }^{1}$ Resident Radiation Oncology, Army Hospital (R\&R), India \\ ${ }^{2}$ Professor Radiation Oncology, Army Hospital (R\&R), India
}

Submission: July 10, 2017; Published: July 12, 2017

*Corresponding author: Colonel Virender Suhag, Professor \& Head Dept of Radiotherapy, Army Hospital (RR) Delhi-110010, Tel: +91-8826804584;

Email: virendersuhag@gmail.com

\begin{abstract}
A substantial number of patients with cancer suffer considerable pain at some point during their disease, and approximately $25 \%$ of cancer patients die in pain [1]. Cancer pain is prevalent, undertreated, and feared by patients with cancer. The prevalence of pain in cancer patients at various stages of the disease range from 38 to $51 \%$, and increases up to $74 \%$ in the advanced and terminal stages. Despite published World Health Organization (WHO) guidelines for pain management, 42 to $51 \%$ of cancer patients receive inadequate analgesia and $30 \%$ receive no analgesics at all $[2,3]$. Reasons for under-treating cancer pain include attitudes of patients, clinicians, and factors associated with healthcare systems. Poorly managed cancer pain is well known to profoundly impact the patient's daily life and interfere with quality of life. To manage pain in oncology patients, clinicians should assess pain during regular follow-up visits using validated pain measurement tools and follow prescribing guidelines, if necessary referring patients with cancer to pain specialists. While much "treatment inertia" exists in cancer pain control, cancer pain can be safely and effectively managed and should be carried out to alleviate suffering and improve outcomes [4]
\end{abstract}

Keywords: Cancer; Pain; Management; Barriers; Assessment

\section{Summary}

Pain is the most debilitating and disastrous experience encountered by cancer patient during their course of illness and mostly remains underreported and undertreated. Cancer pain is still one of the most feared entities in cancer and about $75 \%$ of cancer patients with pain require treatment with opioids for severe pain. The worsening cancer-related chronic pain in survivors has been found to have a negative impact on quality of life. An understanding of the basics of pain pathophysiology, assessment, pharmacologic management, and a familiarity with common pain presentations will allow clinicians to effectively manage pain for these patients. Proper use of therapeutic approaches should result in excellent pain control in nearly 95\% of patients with cancer pain but unfortunately, cancer pain, still remains grossly undertreated throughout the world [5]. Various factors affecting pain management include the institutional setup and the awareness and knowledge of patient, nursing staff and treating physicians [6]. Pain management will be most effective when treatments are individualized after exploring the various physical and non-physical components of pain, and the patient and family are educated and involved in decision making [7].

\section{Causes of Pain In Cancer Patients}

Most cancer pain occurs when a tumor presses on bone, nerves or organs. The pain may vary according to location. The tumor, its loco regional spread and its evaluation contributes for up to $90 \%$ of pain while rest is attributed to other non related pain generators. Out of this $90 \%, 70 \%$ is due to tumor invasion or compressing soft tissue, bone, or neural structures while $20 \%$ of pain is due to procedures related to evaluation or therapeutics. Treatment-related pain involving chemotherapy, radiotherapy and surgery can cause pain. Surgery is a frequent cause of pain in patients with cancer and can consist of biopsy, removal, or debulking of a tumor or management of a complication of the tumor. These procedures are often associated with injury to local nerves and postoperative pain, which can later lead to neuromas and chronic pain syndromes. Surgically induced nerve injuries are most commonly seen after breast cancer surgery, thoracotomy, radical neck dissection, and limb amputation. 
These neuromas and chronic pain syndromes are severe and difficult to manage $[5,8]$.

\section{Assessment by scales / questionnaires}

Adequate cancer pain assessment using valid and reliable tools is essential for proper cancer pain management. Because cancer pain can be a complex phenomenon, assessment of its many domains should be conducted using multidimensional tools. This assessment should include an in-depth interview that explores the multidimensional nature of pain (pain descriptors, associated distress, functional impact, and related physical, psychological, social, and spiritual factors) and captures information about cancer treatment history and co morbid conditions, psychosocial and psychiatric history (including substance use), and prior treatments for the pain. The assessment should characterize the pain, clarify its cause, and make inferences about pathophysiology. Visual analogue scales, verbal and numerical rating scales and some multidimensional tools such as the Brief Pain Inventory (BPI) and the McGill Pain Questionnaire are helpful in the assessment of cancer pain provided the limitations of their validity are considered $[5,9,10]$. The BPI measures both the intensity of pain (sensory dimension) and interference of pain in the patient's life (reactive dimension). Recent literature reveals the move toward translating these and other tools to electronic applications [11].

\section{WHO Three Step Analgesic Ladder}

In its simplest form, it is based on use of Paracetamol or NSAIDs for mild pain (score on numerical rating scale i.e. NRS $<3$ out of 10 ), weak opioids \pm paracetamol or NSAIDs for mild to moderate pain (score on NRS 3-6 out of 10); and strong opioids \pm paracetamol or NSAIDs for moderate to severe pain (score on NRS 3-6 out of 10). The analgesic ladder requires use of right drug in right doses, at right intervals, by the mouth, by the clock, by the ladder, and for the individual. As soon as the pain is assessed minimum opioids dose that provides effective pain relief with minimum side effects is titrated. Though a dose range of five to $1000 \mathrm{mg}$ morphine could be used (due to its lack of ceiling effect) doses higher than $200 \mathrm{mg}$ should prompt physicians to reconsider the diagnosis in favor of a neuropathic or morphine resistant pain and should invite alternative supportive measures like neuro-ablation and opioid rotation for better pain control and lesser toxicity.

The use of adjuvant like anti-emetics, anti-diarrheal, antidepressants, anti-psychotics, anticonvulsants, corticosteroids, psycho-stimulants are available in each step. Since this 3 step ladder arrangement provides pain relief to 77-100 percent of patients, the need for inclusion of interventional modalities as a fourth step in the ladder could not generate clear consensus. An alternative route for opioid administration should be considered when oral administration is not possible because of severe vomiting, bowel obstruction, severe dysphagia or severe confusion, as well as in the presence of poor pain control, which requires rapid dose escalation, and/or in the presence of oral opioid-related adverse effects [5,10,11].

\section{Role of Opioids}

Clinicians may prescribe a trial of opioids in carefully selected cancer survivors with chronic pain who do not respond to more conservative management and who continue to experience pain-related distress or functional impairment. Nonopioid analgesics and/or adjuvants can be added as clinically necessary. Clinicians should incorporate a universal precautions approach to minimize abuse, addiction, and adverse consequences of opioid use such as opioid-related deaths. Clinicians should be cautious in co prescribing other centrally acting drugs, particularly benzodiazepines. The pertinent laws and regulations regarding the prescribing of controlled substances should be understood and adhered to. The patients and family members should be educated regarding the risks and benefits of long-term opioid therapy and the safe storage, use, and disposal of controlled substances. Possible myths and misconceptions about medication use should be addressed and patients should be educated about the need to be cautious when using alcohol or sedating over-the-counter medications or in receiving centrally acting medications from other physicians. If opioids are no longer warranted, clinicians should taper the dose to avoid abstinence syndrome. The rate of tapering and the use of co therapies to reduce adverse effects should be individualized for each patient [10-12].

\section{Neuropathic Pain}

Unrelieved neuropathic pain continues to be a substantial health problem in a cancer patient arises due to tumor infiltration or due to paraneoplastic or treatment-induced polyneuropathy. Neuropathic pain affects $6-8 \%$ of the general adult population. It is reported by $27 \%$ of chronic pain patients and $40 \%$ of cancer patients, yet there is no standardized diagnostic test for neuropathic pain. Neuropathic pain can be overlooked in cancer patients. The advent of screening tools can help in recognizing it. This pain is more severe and debilitating then nociceptive pain. Management of intractable neuropathic pain, that arises from tumour invasion of the meninges, spinal cord and dura, nerve roots, plexuses and peripheral nerves is a challenge, requires indepth knowledge and the formulation of a careful management plan usually multimodal therapy. Accurate establishment of incidence, prevalence, severity, and effectiveness of treatment in cancer induced neuropathies is quite essential to find out the burden of this symptom and requirement of effective resource generation. In cancer patients with neuropathic pain, nonopioid and opioid analgesics may be combined with tricyclic antidepressant drugs or anticonvulsants and subjected to side effects monitoring [10,13-15].

\section{Barriers in Management of Cancer Pain}

The important barriers in adequate management of cancer pain include poor resources in developing countries and 
unavailability of morphine. The income is low in developing countries and the cancer load high and thus the resources for cancer pain alleviation are grossly inadequate. WHO reports that $80 \%$ of cancer patients have no access to opiates. Inadequate pain management can be attributed to barriers related to health care professionals, patients, and the health care system.

Common professional-related barriers include poor pain assessment, lack of knowledge and skill, and the reluctance of physicians to prescribe opioids. Patient-related barriers include cognitive factors, affective factors, and adherence to analgesic regimens. System-related barriers such as limits on access to opioids and the availability of pain and palliative care specialists present additional challenges, particularly in resource-poor regions. Given the multidimensional nature of cancer pain and the multifaceted barriers involved, effective pain control mandates multidisciplinary interventions from interprofessional teams. Educational interventions for patients and health care professionals may improve the success of pain management. Awareness of these disparities in access to care should be considered in the context of this clinical practice guideline, and health care providers should strive to deliver the highest level of cancer care to these vulnerable populations [5,16-18].

\section{Neuro-Ablative Procedures for Pain Relief}

Use of neuroablative procedures in cancer pain relief had improved quality of life in patients when compared to oral analgesics. These neuro-ablative procedures provide better pain relief when used in early stages of cancer. Neuro-ablation is the physical interruption of pain pathways either surgically, chemically or thermally. Neuromodulation is the dynamic and functional inhibition of pain pathways either by administration of opioids and other drugs intraspinally or intraventricularly or by stimulation. The revolutionary success of neuroablative procedures in controlling cancer pain has largely been attributed to improvement in procedural techniques as well as simultaneous use of imaging techniques like fluoroscopy, CT, MRI or Ultrasound guided endoscopic procedures. Some of the commonly performed neuro-ablative procedures are celiac plexus block, endoscopic ultrasound guided celiac plexus neurolysis, superior hypogastric plexus block, ganglion impair block, percutaneous vertebroplasty, percutaneous cordotomy, and pre-sacral neurolytic block for pain relief from pelvic cancer $[5,19]$.

\section{Cancer Pain and Anxiety}

Anxiety and pain can be understood with a multidimensional framework that accounts for somatic, emotional, cognitive, and behavioral aspects of these conditions. Patients with cancer pain and anxiety may cause difficult diagnostic dilemmas because some degree of anxiety is a normal response to having a severe medical illness. Furthermore, the somatic symptoms of anxiety often overlap with symptoms related to underlying disease processes or treatment effects. The degree of disruption in a patient's life often is the critical factor in distinguishing normal from maladaptive anxiety. Making an accurate diagnosis will help guide anxiety treatment and screening instruments can facilitate the recognition of those patients in need of further assessment. The relationship between pain and anxiety is complex and bidirectional, with interactions occurring on physiologic and psychological levels. There are a variety of psychopharmacologic, psychotherapeutic, and complementary/ alternative treatments available. A comprehensive approach to care includes these approaches in an individualized way. Terminal sedation is examined as a compassionate option for relieving intractable distress at the end of life $[5,11]$.

\section{Breakthrough Pain}

Breakthrough pain (BTP) is a transient escalation of pain superadded to a controlled base line pain and occurs in $40-80 \%$ of cancer patients. It has a high intensity, starting spontaneously without patient's activity and rapidly reaches a crescendo. The duration of the pain is short and typically lasts for 30 to 45 minutes. Its presence signifies severe pain, stress, functional inability and poor quality of life. However, oral transmucosal fentanyl, intravenous morphine or sub cutaneous morphine could be helpful in relieving BTP. Recent developments in buccal transmucosal administration of lipophilic (e.g. fentanyl) or unionized drugs for direct absorption into systemic circulation have revealed that these drugs can have transcellular movement. Oral mucosa has large surface area with consistent blood supply and uniform temperature. This favors rapid absorption of lipophilic agents. Hydrophilic agents like morphine follow paracellular route to find their way to systemic circulation $[14,20]$.

\section{Conclusion}

To conclude, mostcancerpatients sufferfrom profound, poorly managed pain during their clinical course, and approximately $25 \%$ of cancer patients die in pain. Providing effective pain management for patients with severe pain that impacts quality of life can present the oncologist or palliative care specialist with complex clinical challenges that often require multifaceted therapeutic measures. Increased understanding of available options for truly effective pain management in the oncology and palliative care arena and the benefits of multidisciplinary cooperation will translate into genuine improvements in patient quality of life and a measurable decrease in the number of patients who suffer needlessly in their final days.

\section{References}

1. Stearns L, Boortz-Marx R, Du Pen S, Friehs G, Gordon M, et al. (2005) Intrathecal drug delivery for the management of cancer pain: a multidisciplinary consensus of best clinical practices. J Support Oncol 3(6): 399-408.

2. MH J van den Beuken-van Everdingen, JM de Rijke, AG Kessels, HC Schouten, et al. (2007) Prevalence of pain in patients with cancer: a systematic review of the past 40 years. Annals of Oncology 18: 14371449. 


\section{Cancer therapy \& Oncology International Journal}

3. Ripamonti C, Zecca E, Brunelli C, Groff L, Boff R, et al. (2000) Pain experienced by patients hospitalized at the National Cancer Institute of Milan: Research Project 'Towards a Pain-Free Hospital'. Tumori 86(5): 412-418.

4. Pergolizzi JV, Gharibo C, Ho KY (2015) Treatment Considerations for Cancer Pain: A Global Perspective. Pain Pract 15(8): 778-792.

5. Thapa D, Rastogi V, Ahuja V (2011) Cancer pain management-current status. J Anaesthesiol Clin Pharmacol 27(2): 162-168.

6. Wadhwa R, Chilkoti G, Saxena AK (2015) Current Clinical Opinions, Attitudes and Awareness of Interns Regarding Post-operative and Cancer Pain Management in A Tertiary Care Centre. Indian J Palliat Care 21(1): 49-55.

7. Dalal S, Bruera E (2012) Assessing cancer pain. Curr Pain Headache Rep 16(4): 314-324.

8. Leppert W, Zajaczkowska R, Wordliczek J, Dobrogowski J, Woron J, et al. (2016) Pathophysiology and clinical characteristics of pain in most common locations in cancer patients. J Physiol Pharmacol 67(6): 787799

9. Kumar SP (2011) Utilization of Brief Pain Inventory as an Assessment Tool for Pain in Patients with Cancer: A Focused Review. Indian J Palliat Care 17(2): 108-115.

10. Ripamonti CI, Bandieri E, Roila F, ESMO Guidelines Working Group (2011) Management of cancer pain: ESMO Clinical Practice Guidelines. Ann Oncol 22(6): 139-154.

11. Paice JA, Lacchetti C, Bruera E (2016) Management of Chronic Pain in Survivors of Adult Cancers: ASCO Clinical Practice Guideline Summary. J Oncol Pract 12(8): 757-762.

12. Fielding F, Sanford TM, Davis MP (2013) Achieving effective control in cancer pain: a review of current guidelines. Int J Palliat Nurs 19(12): 584-591.

13. Goyal A, Bhatnagar S (2014) Neuropathic pain in cancer. Ann Palliat Med 3(1): 1-3.

14. Yamaguchi T, Shima Y, Morita T, Hosoya M, Matoba M et al. (2013) Clinical guideline for pharmacological management of cancer pain: the Japanese Society of Palliative Medicine recommendations. Jpn J Clin Oncol 43(9): 896-909.

15. Pérez C, Sánchez-Martínez N, Ballesteros A, Blanco T, Collazo A, et al. (2015) Prevalence of pain and relative diagnostic performance of screening tools for neuropathic pain in cancer patients: A crosssectional study. Eur J Pain 19(6): 752-761.

16. Meghani SH, Knafl GJ (2017) Salient concerns in using analgesia for cancer pain among outpatients: A cluster analysis study. World J Clin Oncol 8(1): 75-85.

17. Meghani SH, Thompson AM, Chittams J, Bruner DW, Riegel B (2015) Adherence to Analgesics for Cancer Pain: A Comparative Study of African Americans and Whites Using an Electronic Monitoring Device. J Pain 16(9): 825-835.

18. Kwon JH (2014) Overcoming barriers in cancer pain management. J Clin Oncol 32(16): 1727-1733.

19. Klepstad P, Kurita GP, Mercadante S, Sjøgren P (2015) Evidence of peripheral nerve blocks for cancer-related pain: a systematic review. Minerva Anestesiol 81(7): 789-793.

20. Haugen DF, Hjermstad MJ, Hagen N, Caraceni A, Kaasa S, et al (2010) Assessment and classification of cancer breakthrough pain: a systematic literature review. European Palliative Care Research Collaborative (EPCRC) pain 149(3): 476-482

\section{Your next submission with Juniper Publishers} will reach you the below assets

- Quality Editorial service

- Swift Peer Review

- Reprints availability

- E-prints Service

- Manuscript Podcast for convenient understanding

- Global attainment for your research

- Manuscript accessibility in different formats (Pdf, E-pub, Full Text, Audio)

- Unceasing customer service

Track the below URL for one-step submission https://juniperpublishers.com/online-submission.php 